\title{
Corrigendum: Parthanatos mediates AIMP2-activated age-dependent dopaminergic neuronal loss
}

Yunjong Lee, Senthilkumar S Karuppagounder, Joo-Ho Shin, Yun-Il Lee, Han Seok Ko, Debbie Swing, Haisong Jiang, Sung-Ung Kang, Byoung Dae Lee, Ho Chul Kang, Donghoon Kim, Lino Tessarollo, Valina L Dawson \& Ted M Dawson

Nat. Neurosci. 16, 1392-1400 (2013); published online 25 August 2013; corrected after print 1 June 2015

In the version of this article initially published, the image in Figure $2 c$ described as being from 20-month-old transgenic mice was actually from 2-month-old control mice. New representative images showing a wider field of view have been provided for all of Figure 2c, and Figure $2 \mathrm{~d}$ has been replaced by a new quantification performed independently of the original one. This quantification $(n=3)$ yielded a $P$ value for the comparison between the 2 -month-old control and transgenic of $<0.001$, as compared to $<0.01$ in the original. The errors have been corrected in the HTML and PDF versions of the article.
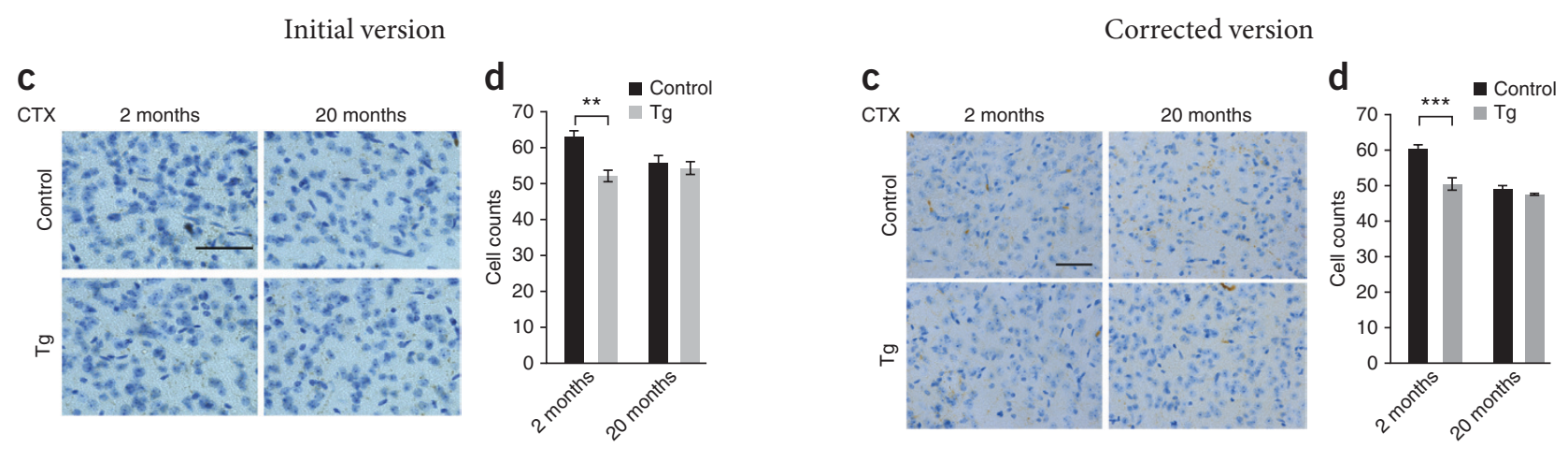

\section{Erratum: Hippocampal-neocortical functional reorganization underlies children's cognitive development}

Shaozheng Qin, Soohyun Cho, Tianwen Chen, Miriam Rosenberg-Lee, David C Geary \& Vinod Menon

Nat. Neurosci. 17, 1263-1269 (2014); published online 17 August 2014; corrected after print 4 June 2015

In the version of this article initially published, the second set of hippocampal coordinates in the fourth Results section was given as $(-26,-22,-1)$. The correct coordinates are $(-26,-22,-16)$. The error has been corrected in the HTML and PDF versions of the article.

Corrigendum: Psychiatric genome-wide association study analyses implicate neuronal, immune and histone pathways

The Network and Pathway Analysis Subgroup of the Psychiatric Genomics Consortium

Nat. Neurosci. 18, 199-209 (2015); published online 19 January 2015; corrected after print 28 April 2015

In the version of this article initially published, the name of author Zhongming Zhao was misspelled Zhaoming Zhao. The error has been corrected in the HTML and PDF versions of the article.

\section{Corrigendum: The dorsal posterior insula subserves a fundamental role in human pain}

Andrew R Segerdahl, Melvin Mezue, Thomas W Okell, John T Farrar \& Irene Tracey

Nat. Neurosci. 18, 499-500 (2015); published online 9 March 2015; corrected after print 26 March 2015

In the version of this article initially published, the labels were reversed for the solid and dotted lines in Figure $2 \mathrm{c}$. The error has been corrected in the HTML and PDF versions of the article. 